\title{
Current status of the Synchrotron Radiation Center
}

\author{
C. J. Moore, ${ }^{a}{ }^{2}$ K. N. Altmann, J. J. Bisognano, R. A. Bosch, D. Eisert, M. Fisher, \\ M. A. Green, R. W. C. Hansen, F. J. Himpsel, H. Höchst, R. L. Julian, K. Kleman, \\ T. Kubala, B. Pedley, G. C. Rogers, M. Severson, J. P. Stott, J. W. Taylor, \\ W. S. Trzeciak, D. J. Wallace, R. Wehlitz, and M. Bissen \\ Synchrotron Radiation Center, University of Wisconsin-Madison, 3731 Schneider Drive, Stoughton, \\ Wisconsin 53589
}

(Presented on 22 August 2001)

The Synchrotron Radiation Center operates the Aladdin electron storage ring at energies of $800 \mathrm{meV}$ or $1 \mathrm{GeV}$ in support of a broad range of national and international research programs with a major focus on the study of valence electrons, spectromicroscopy, and nanolithography. Upgrades to the storage ring have improved the stability of the source, and experiments with low emittance lattice configurations show the feasibility of increased brightness for new or enhanced research. Three recently installed undulators, two pure permanent magnet devices and an electromagnetic device, and the associated instrumentation offer experimentalists high flux combined with high resolution. The status of the existing instrumentation, recent scientific results, and an overview of plans for new undulator-based instruments to cover the photon energy range from 7.8 to $400+\mathrm{eV}$ will be presented. (C) 2002 American Institute of Physics. [DOI: 10.1063/1.1425390]

\section{INTRODUCTION}

The Synchrotron Radiation Center (SRC) at the University of Wisconsin-Madison has been in operation serving the user community for approximately 32 years. First research at the center used the $240 \mathrm{MeV}$ storage ring, Tantalus, which had been originally designed for $\mathrm{e}+/ \mathrm{e}-$ experiments. ${ }^{1}$ This ring supported as many as ten beamlines during the period from 1968 to 1985, operating as the world's first dedicated synchrotron light source.

In 1985 the SRC commissioned its present $1 \mathrm{GeV}$ electron storage ring, Aladdin. Since then, the SRC has striven to satisfy the needs of the user community by providing specialized and upgraded instrumentation, and increasing beam stability and lifetime.

Currently, the focus areas of research at the SRC have been the study of valence electrons and highly correlated systems, cellular spectromicroscopy, and extreme ultraviolet (EUV) lithography. Also, in the last couple of years, the SRC has increased its educational outreach efforts.

\section{THE FACILITY}

The SRC Aladdin electron storage ring is housed in a 21,000 square foot building and consists of 12 bending magnets with three synchrotron radiation ports per magnet for a total of 36 ports. Of these, 21 have monochromators available to users. Each of the four long straight sections is equipped with an undulator. See Fig. 1 for an illustration of the SRC Aladdin electron storage ring.

To facilitate and increase the productivity of the research process at the SRC, ancillary resources are available to users. These include a clean room for sample preparation, a mechanical shop, electronic support, and a variety of

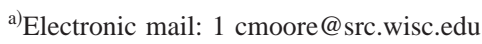

research instrumentation. This instrumentation is maintained by the SRC and allows our user community access to state-of-the-art equipment.

The SRC is currently the home of the Canadian Synchrotron Research Facility and the Center for Nano Technology (CNTech). CNTech makes use of class 100 clean rooms which house the latest steppers and aligners for prototype work in EUV and x-ray lithography.

\section{SOURCE CHARACTERISTICS}

The Aladdin storage ring $(800 \mathrm{MeV}-1 \mathrm{GeV})$ has undergone many upgrades over the years to improve the stability, lifetime, and source size. These include the addition of a fourth harmonic cavity to increase the beam lifetime, ${ }^{2}$ installation of new optical diagnostic monitors to monitor beam quality, and novel rf clearing to reduce ion trapping. Recently, experiments have been performed to implement operation with a lower emittance lattice that will increase the available flux density for users. ${ }^{3}$ Reduction of the horizontal beam size by a factor of 2-4 has been obtained experimentally and can be seen in Fig. 2.

\section{INSERTION DEVICES}

The Aladdin ring has undulators in each of its four long straight sections as summarized in Table I. ${ }^{4}$ They serve as high brightness sources for various research programs and were designed to provide EUV and soft $\mathrm{x}$-ray radiation in the range from 6 to $400+\mathrm{eV}$. This makes the SRC the largest U.S. provider of undulator radiation in the optimized spectral range for probing valence electrons $(\sim 5-50 \mathrm{eV}$ photon energy). The first of these, U2A, was the original Halbachdesigned 30-period pure permanent magnet (PPM) undulator built in $1980 .{ }^{5}$ It was recently replaced by U2B which has $90 \%$ spectral fidelity through the ninth harmonic. The energy 


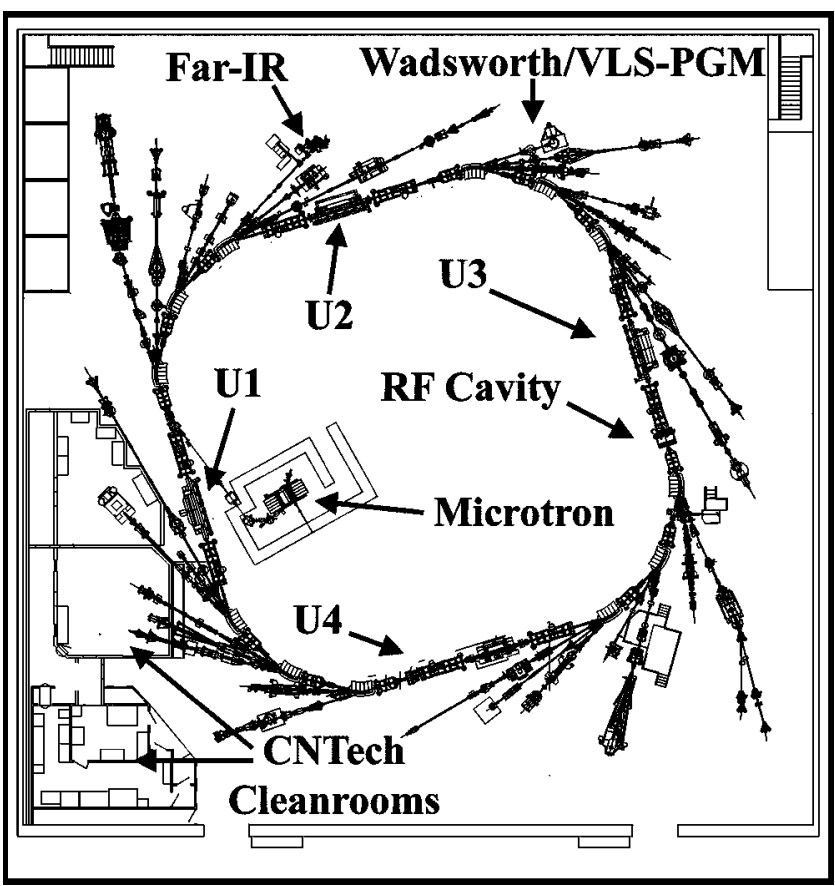

FIG. 1. SRC Aladin storage ring.

ranges shown in Table I encompass both 800 and $1000 \mathrm{MeV}$ operation and the limitations of the associated beamlines. All of the SRC undulators can be energy scanned (with some modest limitations) without disruption to normal operations.

\section{BEAMLINES}

The SRC has 21 beamlines available for researchers. A partial listing can be found in Table II. Following is a description of new undulator beamlines under construction, most of which are low energy and will facilitate the study of valence electrons.

A high resolution $4 \mathrm{~m}$ normal incidence monochromator (NIM) is coupled to the U1 elecgtromagnetic (EM) undulator which covers the range from 6 to $50 \mathrm{eV}$. This beamline is used for the studies of high- $T_{\mathrm{c}}$ superconductors, quantum well states, and high resolution spectroscopy of atomic and molecular orbitals.

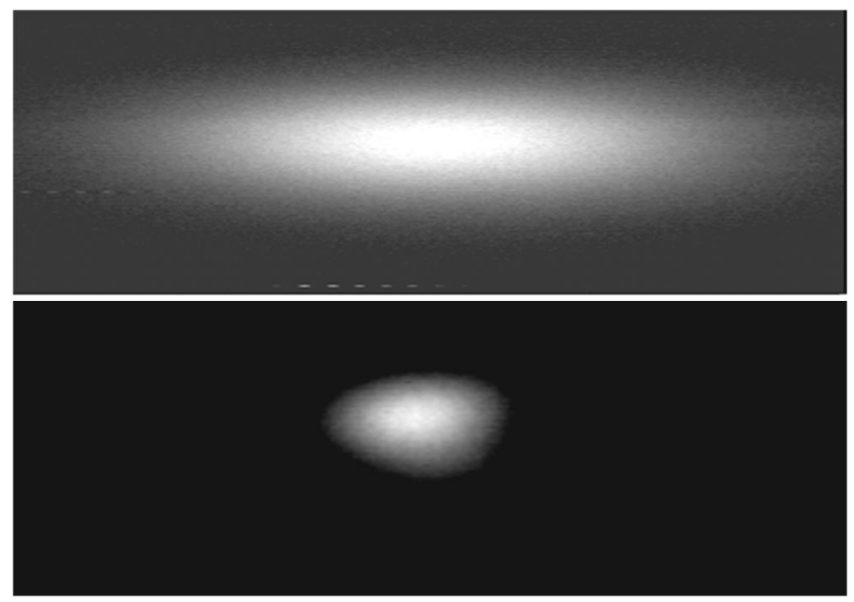

FIG. 2. Images of the SRC Aladdin storage ring electron beam. Normal lattice profile (top) and LF20 lower emittance lattice profile (bottom).
TABLE I. Undulators commissioned at the SRC.

\begin{tabular}{lcccccc}
\hline \hline Name & Year & $\begin{array}{c}\lambda_{u} \\
(\mathrm{~cm})\end{array}$ & $g_{\min }$ & $K_{\max }$ & $N$ & $\begin{array}{c}\text { Range } \\
(\mathrm{eV})\end{array}$ \\
\hline U2A & 1986 & 6.1 & 24 & 1.9 & 30 & $35-150$ \\
$\mathrm{U} 4$ & 1993 & 16 & $35^{\mathrm{a}}$ & 1.8 & 14 & $15-30$ \\
$\mathrm{U} 3$ & 1995 & 7.1 & 24 & 4.6 & 48 & $8-240$ \\
$\mathrm{U} 1$ & 1998 & 10.9 & $24^{\mathrm{a}}$ & 4.5 & 30 & $6-50$ \\
U2B & 2000 & 6.8 & 24 & 4.6 & 51 & $7.8-350^{\mathrm{b}}$ \\
\hline \hline
\end{tabular}

${ }^{\mathrm{a}} \mathrm{EM}$ fixed gap.

${ }^{\mathrm{b}}$ Design.

A new high flux Wadsworth monochromator is currently under construction and will be coupled to the new Danfysik pure permanent magnet undulator U2B. This beamline will cover the energy range from 7.8 to $50 \mathrm{eV}$ and is optimized for studies of magnetic materials using spin-polarized photoemission. The EUV branch line on U2B will focus on research of new photoresist materials for use in the next generation of lithography and micromachining. Also, a third branch line is being developed for U2B which will use a varied line space plane grating monochromator (VLS-PGM) to access photon energies from 50 to $400+\mathrm{eV}$ for use in biological and environmental research.

A plane grating monochromator (PGM) uses the U3 PPM undulator from Maxwell Brobeck to cover the energy range from 8 to $240 \mathrm{eV}$ with high resolution and high throughput and is used primarily for solid state and gas phase photoemission spectroscopy. In addition, the PGM branch line is equipped with a quadruple reflection polarizer that has been shown to deliver a high degree of circular polarization $(>92 \%$ at $21 \mathrm{eV})$ and is used for variable polarization studies. $^{6}$

The U4 EM undulator is coupled to a modified Wadsworth-type monochromator and designed specifically for use in high- $T_{c}$ research, charge density wave materials, and low dimensional solids.

Under construction is a branch line to the existing infrared beamline which uses edge radiation from a bending

TABLE II. List of SRC beamlines.

\begin{tabular}{lc}
\hline \hline Beamlines & $\begin{array}{c}\text { Energy range } \\
(\mathrm{eV})\end{array}$ \\
\hline Undulator beamlines $^{\mathrm{U}}$ 1 NIM & \\
$\mathrm{U} 2 \mathrm{Wadsworth}^{\mathrm{b} *}$ & $6-50$ \\
$\mathrm{VLS}^{\mathrm{P}} \mathrm{PGM}^{\mathrm{b} *}$ & $7.8-50$ \\
$\mathrm{U} 3 \mathrm{PGM}^{\mathrm{c}}$ & $50-400+$ \\
$\mathrm{U} 4 \mathrm{Wadsworth}^{\mathrm{d}}$ & $8-240$ \\
Bending magnet beamlines $^{\mathrm{N}}$ & $15-30$ \\
HERMON $^{\mathrm{f}}$ & \\
IR $^{\mathrm{f}}$ & $245-1100$ \\
Other beamlines $^{\mathrm{g}}$ & $0.01-1$ \\
\hline \hline
\end{tabular}

*Work in progress.

${ }^{\mathrm{a}}$ Reference 12.

${ }^{\mathrm{b}}$ Reference 13 .

${ }^{\mathrm{c}}$ References 14 and 15.

${ }^{\mathrm{d}}$ Reference 16.

${ }^{\mathrm{e}}$ Reference 17.

${ }^{\mathrm{f}}$ Reference 18 .

${ }^{g}$ Reference 19 . 
magnet. ${ }^{7}$ This beamline is optimized for the region of 200$700 \mathrm{~cm}^{-1}$ and will permit far-infrared spectroscopy on samples grown and characterized in a modern UHV vacuum chamber.

\section{RESEARCH HIGHLIGHTS}

The SRC provides users with access to up-to-date sophisticated instrumentation for their research. New instrumentation includes a Scienta SES 200 and SES 2002 analyzers devoted to surface science research programs. The Scienta analyzers are $200 \mathrm{~mm}$ hemispherical analyzers designed for very high resolution electron spectroscopy. The new Scienta 2002 has an energy resolution of $<2 \mathrm{meV}$ and an angular resolution of $0.2^{\circ}$ over a large kinetic energy range. Other instrumentation includes a magnetic circular dichroism chamber for element specific measurements of magnetism systems, a spin-polarized photoemission chamber for determining direct information about the spin of photoelectrons, and an x-ray absorption chamber.

Research at the SRC in the spectromicroscopy area utilizes a photoelectron emission spectromicroscope (PEEM) system developed by researchers at the SRC and a new PEEM system capable of $5.5 \mathrm{~nm}$ spatial resolution that will be upgraded with an aberration correction system allowing spatial resolution down to approximately $1 \mathrm{~nm}$. With these systems researchers have helped to demonstrate that sulfur reducing bacteria can couple their metabolism to the formation of metal sulfides. ${ }^{8}$ Using synchrotron light to detect sulfur in particles associated with the bacteria, researchers concluded that the bacteria cause the precipitation of nanocrystals of zinc sulfide. Other exciting work in the biological arena includes research into the origin of life on earth through studies of the primordial atmosphere contained in geological inclusions.

The EUV lithography program spearheaded by CNTech is currently using the U2B undulator to conduct resist studies and are beginning with exposures at 157 and $13.4 \mathrm{~nm}$. Scientists with CNTech have used X-rays from the Aladdin storage ring to leapfrog the expected next-generation lithographic processes by demonstrating the printing of 50-30 $\mathrm{nm}$ features. ${ }^{9}$ They have developed a method to improve the use of phase masks to enhance the intensity of the bright peak where the feature on the mask is larger, by a factor of $5-6$, than the feature to be printed on the wafer.

Several groups at the SRC have been in the forefront of high- $T_{\mathrm{c}}$ research. Angle resolved photoemission has provided information about the momentum dependence of the electronic states and the superconducting gap, which cannot be obtained by other methods. ${ }^{10}$ This type of research demonstrates the power of the Scienta analyzer with energy and angle multidetection in resolving such complex features in energy and momentum.

An additional benefit to doing research at the SRC is that each user is allocated up to three weeks of beamtime allowing sufficient time for careful sample preparation and char- acterization. This gives researchers a change to perform difficult experiments and has, for example, led to publications that involve artificial structures made with atomic precision. Recently, a connection between the thermal stability of a metallic film and its electronic structure was found in studies of Ag films supported on Fe substrates. These studies depend on the growth of atomically uniform films and sample characterization by photoemission during the preparation process and could not be accomplished in a short experimental period. ${ }^{11}$

\section{EDUCATIONAL ACTIVITIES}

The SRC has increased its efforts in the outreach area. We have conducted yearly open houses, been involved with science summer camps, and participated in activities that help to fulfill our goals of increasing the awareness and understanding of science and science research to students and the general public.

\section{ACKNOWLEDGMENTS}

We gratefully acknowledge the continuing financial support of the National Science Foundation under Award No. DMR-0084402.

${ }^{1}$ D. W. Lynch, J. Synchrotron Radiat. 4, 334 (1997).

${ }^{2}$ K. J. Kleman, in Proceedings of the 1995 Particle Accelerator Conference, Dallas, 1995, p. 1785.

${ }^{3}$ J. J. Bisognano, R. A. Bosch, D. E. Eisert, M. A. Green, K. J. Kleman, and W. S. Trezeciak, in Proceedings of the 2001 Particle Accelerator Conference, Chicago, June 18-22, 2001 (to be published).

${ }^{4}$ M. A. Green, W. S. Trzeciak, D. E. Eisert, R. A. Bosch, J. J. Bisognano, D. J. Wallace, G. C. Rogers, and M. V. Fisher, in Ref. 3.

${ }^{5}$ W. S. Trzeciak, R. W. C. Hansen, M. A. Green, M. Bissen, and D. Eisert, Nucl. Instrum. Methods Phys. Res. A 291, 413 (1990).

${ }^{6}$ A. Kaminski (private communication).

${ }^{7}$ R. W. C. Hansen, R. Julian, T. Kubala, R. A. Bosch, T. E. May, and C. Hirschmugl, these proceedings.

${ }^{8}$ M. Labrenz et al., Science 290, 1744 (2000).

${ }^{9}$ L. Yang, F. Cerrina, and J. W. Taylor, J. Vac. Sci. Technol. (submitted).

${ }^{10}$ A. Kaminski et al., Phys. Rev. Lett. 84, 1788 (2000).

${ }^{11}$ D.-A. Luh, T. Miller, J. J. Paggel, M. Y. Chou, and T.-C. Chiang, Science 292, 1131 (2001).

${ }^{12}$ T. Kubala, M. Bissen, M. Severson, G. Rogers, D. Wallace, M. Thikim, and M. V. Fisher, in Proceedings of the SRI: Eleventh US National Conference AIP Conf. Proc., No. 521, edited by P. Pianetta, J. Arthur, and S. Brennan (AIP, New York, 2000), p. 91.

${ }^{13}$ J. Bisognano, M. Severson, M. A. Green, G. Rogers, M. Fisher, T. Kubala, and M. Bissen, Nucl. Instrum. Methods Phys. Res. A 467, 492 (2001).

${ }^{14}$ R. Reininger, S. L. Crossley, M. A. Lagergren, M. C. Severson, and R. W. C. Hansen, Nucl. Instrum. Methods Phys. Res. A 347, 304 (1994).

${ }^{15}$ F. C. Younger, W. J. Pearce, and B. Ng, Nucl. Instrum. Methods Phys. Res. A 347, 98 (1994).

${ }^{16}$ C. G. Olson, and D. W. Lynch, Nucl. Instrum. Methods Phys. Res. A 347, 278 (1994).

${ }^{17}$ M. Bissen, M. Fisher, R. Reininger, G. Rogers, and H. Höchst, in SRI: Proceedings of the Tenth U.S. National Conference, AIP Conf. Proc., No. 417, edited by Ernest Fontes (AIP, New York, 1997), pp.17-21.

${ }^{18}$ T. E. May, R. A. Bosch, and R. L. Julian, in Proceedings of the 1999 Particle Accelerator Conference, New York, 1999, p. 2394.

${ }^{19}$ D. L. Huber, M. A. Green, R. Hansen, H. Höchst, R. Reininger, E. Rowe, W. Trzeciak, and B. P. Tonner, Nucl. Instrum. Methods Phys. Res. A 347, 49 (1994). 